Publications of the Astronomical Society of the Pacific, 110:732-737, 1998 June

(C) 1998. The Astronomical Society of the Pacific. All rights reserved. Printed in U.S.A.

\title{
VIRIS: A Visual-Infrared Imaging System for the Lick Observatory 1 Meter Telescope
}

\author{
James R. Graham and Lynne A. Hillenbrand \\ Department of Astronomy, 601 Campbell Hall, University of California, Berkeley, CA 94720; jrg@astro.berkeley.edu, lah@astro.caltech.edu \\ AND \\ Anthony A. Misch \\ UCO/Lick Observatory, University of California, Santa Cruz, CA 95064; tony@ucolick.org \\ Received 1998 January 5; accepted 1998 February 16
}

\begin{abstract}
We describe a system in use at the Lick Observatory $1 \mathrm{~m}$ Nickel telescope for near-simultaneous imaging at optical and near-infrared wavelengths. The combined availability of a CCD and a NICMOS3 camera makes the system well suited for photometric monitoring from 0.5 to $2.2 \mu \mathrm{m}$ of a variety of astrophysical objects. Our science program thus far has concentrated on studying variability trends in young stellar objects.
\end{abstract}

\section{INTRODUCTION}

There is broad need in astronomy for simultaneous opticalinfrared photometric capabilities that enable temporal monitoring. Examples include recording supernova light curves, monitoring variability of active galactic nuclei (AGNs), and measuring the spectral energy distributions of young stars. Because supernovae are standard candles, their light curves are important and have maximum utility if they are well sampled and cover multiple wavelengths. Dense temporal sampling is valuable because this helps to establish the epoch of maximum light, a task that is simplified for type Ia events if $J$-band data are available, and some immunity to the effects of dust can be achieved if observations extend into the $K$ band, where extinction is 1 order of magnitude lower than at visible wavelengths (Elias \& Frogel 1981). Variability of AGNs such as quasars and Seyfert nuclei provides an opportunity to investigate the nature of the central engine, and the distribution and properties of dust grains via reverberation mapping (see, e.g., Nelson 1996). Part of the data for Nelson's (1996) study came from a dual optical-IR imager on the UCLA 24 inch $(0.6 \mathrm{~m})$ telescope (Nelson et al. 1997). Another example of a simultaneous optical-IR system is the $I J K$ camera used for the DENIS survey (Epchtein et al. 1994).

Our primary interest thus far has been to pursue an opticalinfrared photometric monitoring program to investigate and characterize variability trends in the spectral energy distributions (SEDs) of young stars. Circumstellar accretion disk models matched to the observed SEDs of young pre-main-sequence stars are used to attempt understanding of the complex accretion processes in these systems. A major uncertainty in the SED modeling procedure, however, is the continuum variability,

\footnotetext{
${ }^{1}$ Current address: Division of Physics, Math, and Astronomy, California Institute of Technology, Pasadena, CA 91125.
}

which occurs at all wavelengths out to at least $3.5 \mu \mathrm{m}$. Changes in flux of several tens to several hundreds of percent occur on timescales of a few hours to a few days, depending on the source and on the wavelength regime. There are several possible causes for the observed monochromatic and color variability: (1) variable stellar flux; (2) variable extinction; and (3) variable properties of the circumstellar material. Sufficient optical and near-infrared monitoring has been carried out for a few sources, so that we are able to investigate their complicated behavior in color-color and color-magnitude diagrams (cf. Skrutskie et al. 1996; Herbst, Herbst, \& Grossman 1994). These patterns seem inconsistent with simple variability explanations like changes in intrinsic stellar flux or line-of-sight obscuration (although these causes cannot yet be discounted entirely). Thus, it may be that the observed variability trends are due to changes in the physical properties of the hot circumstellar gas and dust, e.g., temperature, opacity, or geometry variations taking place in accretion columns, in the inner disk, or in the stellar/disk wind. Testing this hypothesis requires a more extensive photometric data set than currently exists. Relevant monitoring timescales might encompass the dynamical time for a wind $\left(R_{*} / v_{\text {wind }} \sim \mathrm{hr}\right)$, the free-fall time from the inner disk to the stellar surface $\left[\left(r^{3} / G M\right)^{1 / 2} \sim\right.$ tens of $\left.\mathrm{hr}\right]$, the dynamical time for the disk $\left[1 / \Omega=\left(r^{3} / G M\right)^{1 / 2} \sim\right.$ tens of hr to yr for $r=0.1-1$ $\mathrm{AU}]$, the dust destruction/formation time ( $\sim \mathrm{yr})$, and the viscous accretion timescale $\left(\alpha^{-1} r^{2} / h^{2} \tau_{\text {rot }} \gtrsim\right.$ tens of yr).

Both scientific and practical considerations shaped our plans for implementing simultaneous optical-infrared measurements at the University of California's Lick Observatory on Mount Hamilton. Our need to image complex fields with multiple and sometimes extended sources ruled out a single-channel photometer. However, as cost precluded development of new cameras, combining existing instruments to meet our broadband imaging requirements presented an attractive design strategy. 


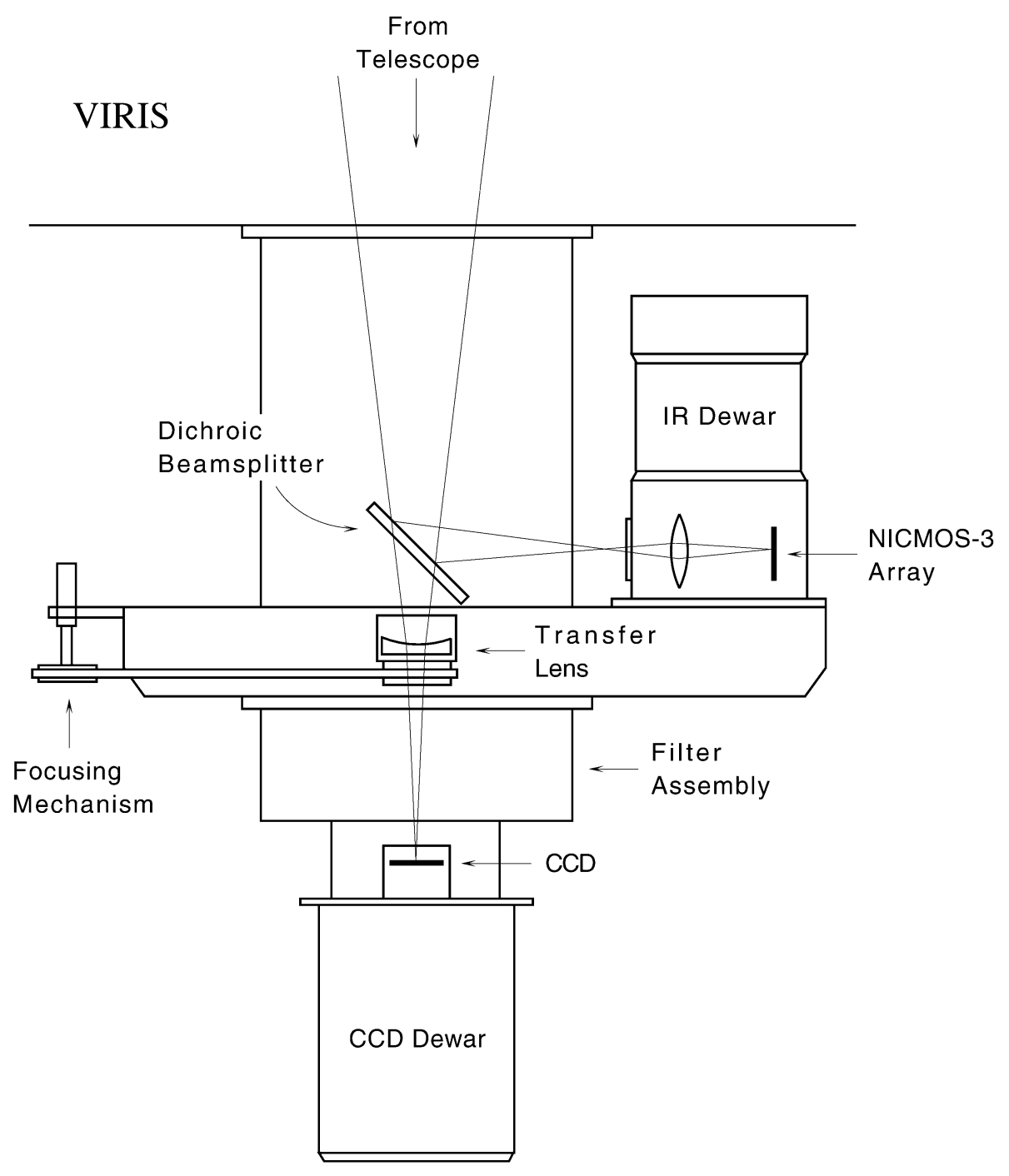

FIG. 1.-Figure showing the location of CCD-2 and LIRC-2 on the Lick $1 \mathrm{~m}$ telescope with the VIRIS interface and transfer lens

This approach also permitted us to take advantage of existing electronic and software controls and to adopt existing mechanical telescope interfaces with only minor modifications.

Since the science goals require intensive photometric monitoring of relatively bright targets, we concentrated our instrument development effort on the $1 \mathrm{~m}$ Nickel telescope, rather than the heavily oversubscribed $3 \mathrm{~m}$ Shane telescope (though, in principle, VIRIS could be used with either). We thus arrived at the option of combining Lick's $1 \mathrm{~m}$ facility CCD camera and the facility IR camera, used at both the 1 and $3 \mathrm{~m}$ telescopes.

\section{OPTICAL AND MECHANICAL CONFIGURATION}

VIRIS is an optomechanical system for combining the Lick Observatory facility NICMOS3 camera, LIRC-2 (Gilmore, Rank, \& Temi 1994), with a standard CCD Dewar containing a $2048 \times 2048$ detector with $15 \mu \mathrm{m}$ pixels, manufactured by Orbit, and an interface box that includes automated filter and aperture wheels (Fig. 1). The use of an optical/IR dichroic beam splitter and a transfer lens delivers parfocal optical and IR images to the respective Dewars and, hence, provides the opportunity to perform simultaneous imaging in the optical and infrared.

The LIRC-2 telescope interface mounts the side-looking infrared camera Dewar on the 1 or $3 \mathrm{~m}$ telescopes at Lick Observatory. The beam from the secondary is relayed to the IR camera by a $45^{\circ}$ IR reflecting gold dichroic beam splitter. In the original version of the LIRC-2 interface, optical light $(\lambda<1 \mu \mathrm{m})$ passes through the dichroic to a bore-sight CCD acquisition and guide camera. This configuration suggested that the acquisition and guide camera could be replaced with a science grade CCD. The existence of an alternate acquisition/ 
guide camera (the TUB) located upstream of the dichroic in the light path means these capabilities are not lost by the replacement with the CCD.

LIRC-2 reimages the telescope focal plane from a position about $50 \mathrm{~mm}$ upstream of the Dewar window onto a NICMOS3 detector; hence, the focal plane lies only $200 \mathrm{~mm}$ behind the dichroic, and it is not possible to locate the CCD interface box close enough to this mirror to get simultaneous in-focus images on IR and optical detectors. We decided that the most economical way to achieve this was to locate a diverging lens in the beam, just below the dichroic. This solution throws the focal plane back, while adjustment of the lens position provides a mechanism so that the IR camera and the CCD can be in focus simultaneously. The lens we decided to use is a stock plano-concave antireflection coated borosilicate glass (BK7) singlet with a $-125 \mathrm{~mm}$ focal length from Edmunds Scientific Catalog (part number M 45039). This transfer lens throws the optical focus back by a nominal $140 \mathrm{~mm}$.

In normal operation with LIRC-2 the back focal length of the telescope is increased by displacing the secondary from its nominal position for a classical Cassegrain configuration by 35 $\mathrm{mm}$ toward the primary. This introduces spherical aberration, which is partially compensated for at the optical focus by orienting the transfer lens with its concave surface toward the secondary. The dominant residual aberration of the telescope and transfer lens combination is lateral color amounting to 0.3 for the $R$-band filter at a field angle of $1^{\prime}$. Typical seeing at the $1 \mathrm{~m}$ telescope is about 1".5 FWHM, and therefore this aberration is negligible. The disadvantage of this configuration is that the effective focal length at the CCD is increased by a factor of 2.77 , and a $15 \mu \mathrm{m}$ pixel projects to 0.064 on the sky. With this small pixel scale, the readout is rebinned on-chip $4 \times 4$ to yield a $512 \times 512$ array of 0 ".26 pixels with a square field of view of $2 ! 2 \times 2 ! 2$, which matches the IR field well. Focus differences between $V, R$, and $I$ filters amount to no more than 0.2 of image blur, and refocusing between wavelengths is unnecessary.

The transfer lens is mounted in a finely threaded lens barrel and is coupled to a micrometer head via a toothed timing belt, permitting a total travel of $25 \mathrm{~mm}$; the corresponding motion of the focal plane is $170 \mathrm{~mm}$, and hence this assembly permits easy access to adjust the lens as well as a reproducible way of recording the focus setting. Focusing the lens makes the optical and IR cameras parfocal. Subsequent focus variations, primarily due to thermal expansion and contraction of the telescope truss, henceforth are handled by changing the secondary mirror focus without further need to adjust the transfer lens. Experience observing with VIRIS has shown that the transfer lens focus position is determined once at the beginning of a run and thereafter requires no further adjustment.

The LIRC-2 dichroic has a thick gold coat; it was designed to minimize infrared reflection losses while having sufficient transmission to permit bore-sight guiding at visible wavelengths. The transmission losses due to the dichroic are 2.5 mag at $V, 3.5 \mathrm{mag}$ at $R$, and $4.0 \mathrm{mag}$ at $I$. Since many of our targets are highly extincted and faint at optical wavelengths, the low efficiency of the dichroic added unacceptably large observing overheads. One option would be to replace the dichroic with one that has a thinner gold coat. This choice was unacceptable, since substantially improved transmission at $I$ band could only be achieved by sacrificing reflectivity at $J$. High-performance, multilayer, dielectric beam splitters are available, but they are expensive. We decided that removing the dichroic from the optical path, by locating it on a remotely controllable translation stage, would provide the most satisfactory solution. This scheme has the added advantage to regular users of LIRC-2 of improving the sensitivity of the boresight camera to aid the identification of optically faint targets. Thus, although truly simultaneous optical and infrared observations are feasible (but see $\S 3$ ), our data have been obtained sequentially, with movement of the dichroic out of and into the beam for the optical and infrared observations, respectively. Furthermore, the need for short exposures and dithering in the IR make genuine parallel optical-IR impractical. We did not attempt to make the bore sights of the optical and IR cameras coincide exactly since any offset can be accommodated by a small telescope motion.

\section{ELECTRONICS AND SOFTWARE}

For operation with VIRIS, the CCD camera and LIRC-2 retain their original control electronics and Lick data acquisition systems. The CCD uses a Lick in-house array controller with its interface running on an Integrated Solutions 68030-based workstation. LIRC-2 uses a Leach controller run from a Sun Microsystem Sparc-5. Both machines run UNIX and X-11 and are fully networked.

Both data acquisition systems obtain telescope status information for their respective image headers by querying the telescope control computer. If the optical and infrared arrays are read out simultaneously, a potential race condition can develop. At present, to avoid this conflict for truly simultaneous observations, access to telescope information must be disabled for one of the arrays. This is easily done in software but costs some header information. However, since our observations are sequential rather than simultaneous, this step has not been necessary.

\section{CALIBRATION AND OBSERVING STRATEGY}

Since the dichroic displaces the optical beam on the CCD by about $30^{\prime \prime}$, the optical bore sight moves relative to the IR beam depending on whether or not the dichroic is in the beam for optical observing. To take account of this, an offset is programmed into the observing scripts so that a telescope motion is commanded from the CCD data acquisition system to jog between the optical and IR beams when observing switches from CCD-2 to LIRC-2. 
V
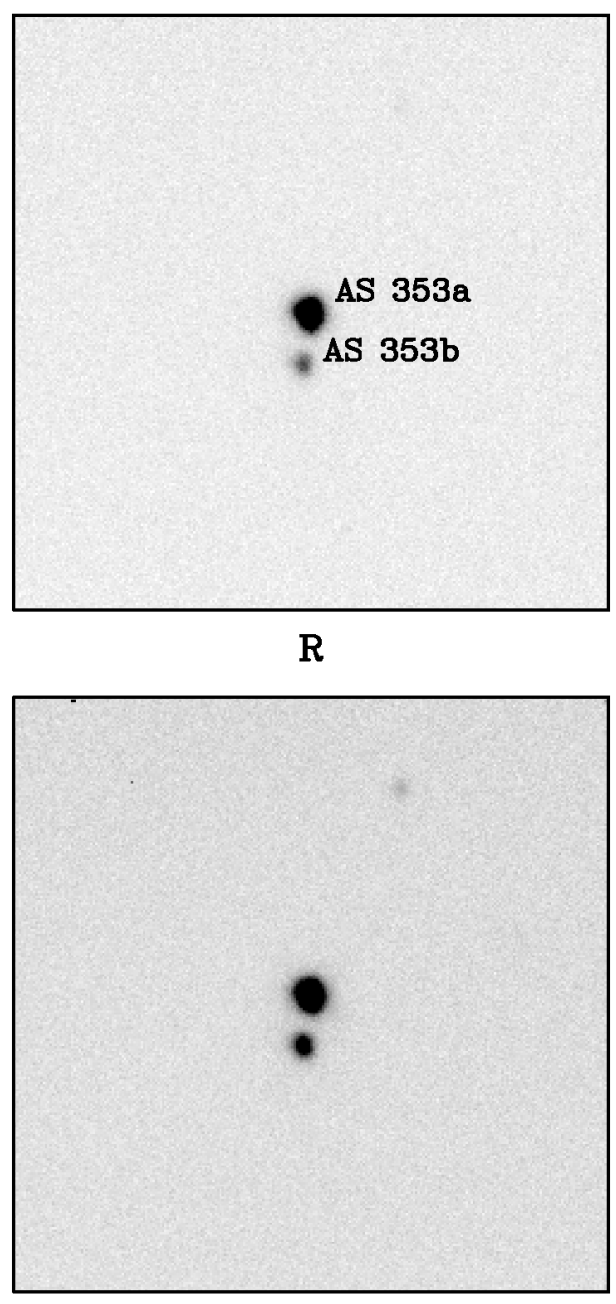

I

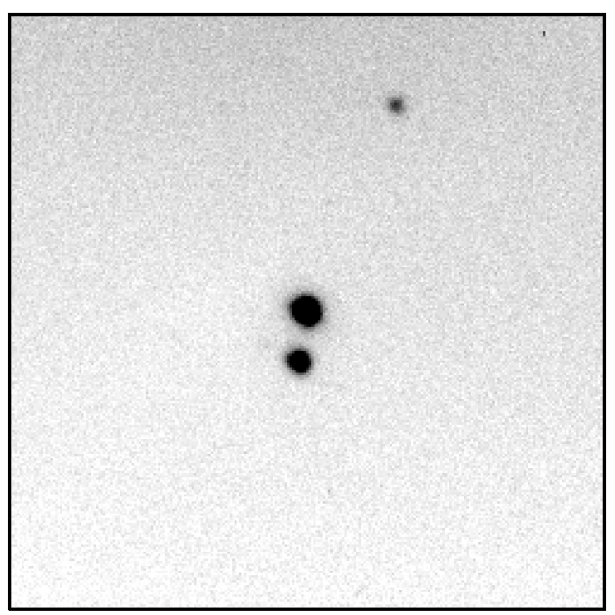

J

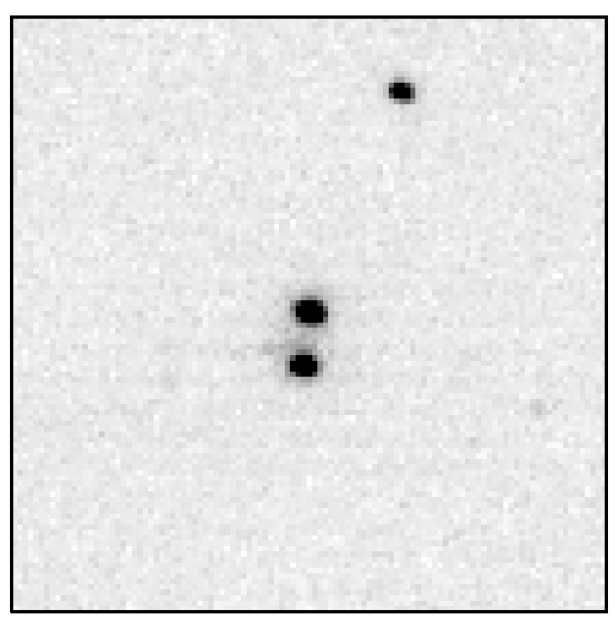

$\mathrm{H}$

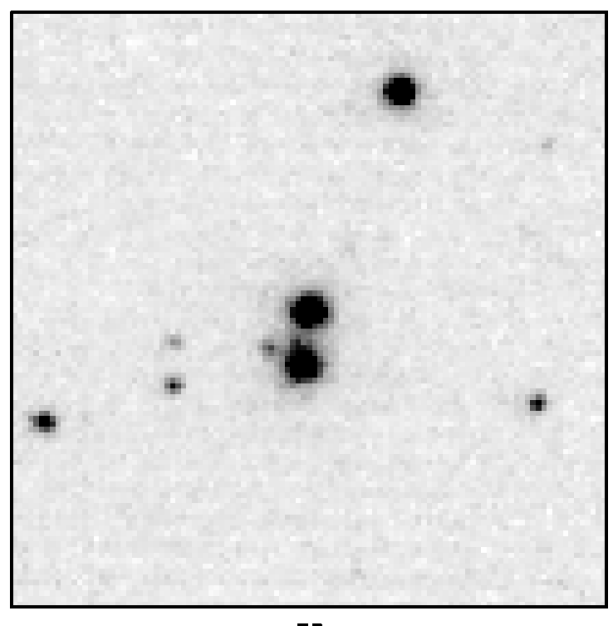

K

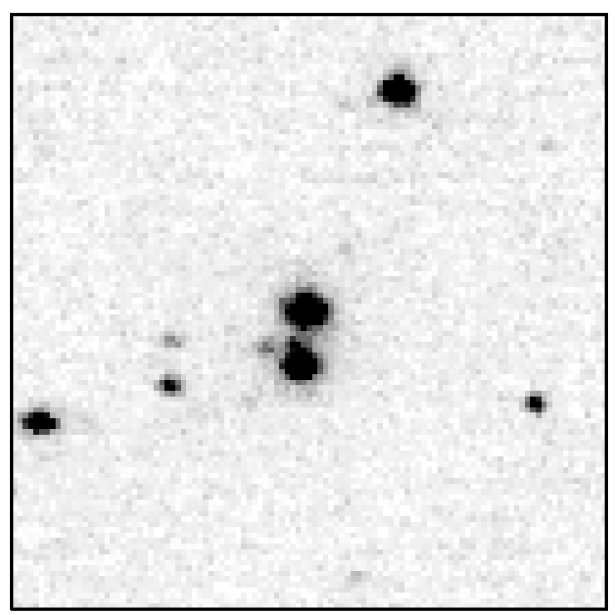

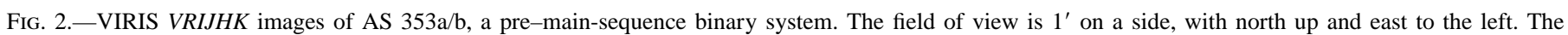
plate scale is 0.25 pixel $^{-1}$ for the optical images and 0.57 pixel $^{-1}$ for the infrared images. Typical seeing at the Lick Observatory 1 m telescope is $\sim 1$ ".5. 
TABLE 1

VIRIS Photometric Zero Points on the $1 \mathrm{~m}$ Telescope ${ }^{\text {a }}$

\begin{tabular}{ccc}
\hline \hline Band & Dichroic Out & Dichroic In \\
\hline$V \ldots \ldots \ldots \ldots \ldots \ldots \ldots$ & 22.4 & 19.9 \\
$R_{\mathrm{C}} \ldots \ldots \ldots \ldots \ldots \ldots \ldots$ & 22.8 & 19.3 \\
$I_{\mathrm{C}} \ldots \ldots \ldots \ldots \ldots \ldots \ldots$ & 23.5 & 19.5 \\
$J \ldots \ldots \ldots \ldots \ldots \ldots \ldots$ & $\ldots$ & 21.1 \\
$H$ & $\ldots \ldots \ldots \ldots \ldots \ldots$ & 21.1 \\
$K^{\prime} \ldots \ldots \ldots \ldots \ldots \ldots \ldots$ & $\ldots$ & 20.8 \\
\hline
\end{tabular}

${ }^{a}$ The zero point is defined as the stellar magnitude that gives an integrated signal of one photoelectron per second. At the time of these observations the gain of the CCD system $\left(V R_{\mathrm{C}} I_{\mathrm{C}}\right)$ was measured to be $6 e^{-}$per $\mathrm{ADU}$, and that of the IR system $(J H K)$ was $9 e^{-}$per ADU.

VIRIS was initially deployed with an oversized transfer lens. An unacceptably high level of scattered light, caused by a reflection between the CCD Dewar and the periphery of the transfer lens, initially made flat fields difficult to measure. In subsequent runs the installation of an aperture stop at the lens, and application of black material to reflective $\mathrm{Al}$ ring supporting the Dewar window, eliminated this reflection and permitted measurement of accurate flat fields. The motion of the translation stage is sufficiently reproducible so that no observable change in flat fields, e.g., due to dust specks on the dichroic, has been detected.

Our observational campaign has concentrated on $\mathrm{T}$ Tauri stars, which are typically red objects, requiring moderate optical exposures 20-300 s (dichroic out) and shorter (5-150 s) IR exposures. The IR exposures consist of five individual, short (1-30 s), dithered frames so that the IR bright sky does not saturate the detector and to permit good sky subtraction. During a typical summer night of $9 \mathrm{hr}$ we can measure some 15-20 target sources in VRIJHK, together with 4-6 photometric standard fields. We primarily use stars common to the Landolt (1983, 1992) optical standards lists and the Casali \& Hawarden (1992) infrared standards list for photometric calibration. Table 1 lists the photometric zero points for VIRIS. These data were derived from several observations of HD 18881. The $V R_{\mathrm{C}} I_{\mathrm{C}}$ data are listed for both positions of the dichroic.

\section{SCIENCE RESULTS}

An example set of VIRIS images is shown in Figure 2, with a limited time-series spectral energy distribution shown in Figure 3. The young stars we have chosen for investigation are all less than $1-2$ Myr of age, $\sim 0.5-3 M_{\odot}$ in mass, and have demonstrated optical and near-infrared variability from data in the literature.

Our strategy has been to observe the same list of targets every night of every 3-5 night run with $2-4$ weeks separating runs. Thus far, about 30 nights of photometry have been collected over two summer seasons (1996 and 1997). Our sampling is not good enough to search for rotation periods, but we can correlate optical and infrared monochromatic and color variability. With the construction of accurate SEDs (i.e., based

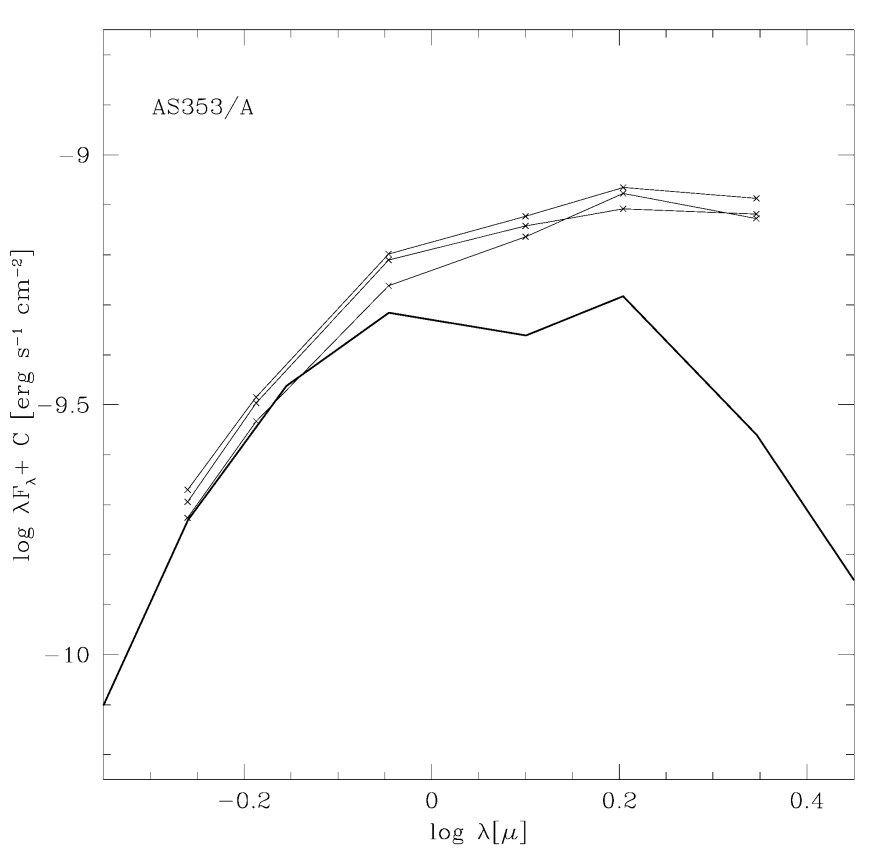

FIG. 3.-Spectral energy distribution of AS 353a from 0.55 to $2.2 \mu \mathrm{m}$. Three consecutive nights of photometry from VIRIS are shown (crosses connected by light solid lines) along with the spectral energy distribution of an unreddened K5 star (heavy solid line), for comparison. Error bars are not shown since they would be smaller then the plotted symbols. The comparison star has been arbitrarily normalized at $V$ to our faintest measurement. Note the flux variability at all wavelengths and the large amount of excess emission in the near-infrared.

on near-simultaneous optical and infrared data), and an understanding of their variability trends, our eventual goal is to investigate whether changes in some combination of the geometry of the circumstellar dust and the accretion rate, can account for the observed flux variability in these young stars.

\section{CONCLUSIONS}

We have designed and constructed an efficient system for near-simultaneous optical and IR imaging and used it to obtain accurate VRIJHK photometry in a program to monitor the variability of T Tauri stars. VIRIS demonstrates the feasibility of combining existing optical and IR instruments using an optomechanical interface to permit simultaneous and near-simultaneous operation. We have shown that the details of the interface are very simple, and likely could be replicated on other small telescopes to permit them to tackle similar scientific programs that require panchromatic observations.

We extend our thanks for invaluable assistance from the staff at Lick, both in Santa Cruz and on Mount Hamilton, especially to Matt Radovan and Rem Stone. Support for this program was provided by Joe Miller, the Director of Lick Observatory. J. R. G. is supported in part by a Fellowship from the Packard Foundation. Support to L. A. H. for this 
work was provided by NASA through grant HF1060.01-94A from the Space Telescope Science Institute, which is op- erated by the Association of Universities for Research in Astronomy, Inc., under NASA contract NAS5-26555.

\section{REFERENCES}

Casali, M. M., \& Hawarden, T. G. 1992, UKIRT/JCMT Newsletter, 4, 33

Elias, J. H., \& Frogel, J. A. 1981, ApJ, 251, L13

Epchtein, N., et al. 1994, Ap\&SS, 217, 3

Gilmore, D. K., Rank, D. M., \& Temi, P. 1994, Proc. SPIE, 2198, 744

Herbst, W., Herbst, D. K., \& Grossman, E. J. 1994, AJ, 108, 1906
Landolt, A. U. 1983, AJ, 88, 439 . 1992, AJ, 104, 340

Nelson, B. O. 1996, ApJ, 465, L87

Nelson, B., McLean, I. S., Henriquez, F., \& Magnone, N. 1997, PASP, 109,600

Skrutskie, M. F., Meyer, M. R., Whalen, D., \& Hamilton, C. 1996, AJ, 112, 2168 